\title{
Elastic Chain in a Random Potential: Simulation of the displacement function $\left\langle(u(x)-u(0))^{2}\right\rangle$ and relaxation.
}

\author{
Steven Spencer and Henrik Jeldtoft Jensen \\ Department of Mathematics, Imperial College \\ 180 Queen's Gate, London SW7 2BZ \\ United Kingdom
}

February 3, 2017

\begin{abstract}
We simulate the low temperature behaviour of an elastic chain in a random potential where the displacements $u(x)$ are confined to the longitudinal direction $(u(x)$ parallel to $x$ ) as in a one dimensional charge density wave-type problem. We calculate the displacement correlation function $g(x)=\left\langle(u(x)-u(0))^{2}\right\rangle$ and the size dependent average square displacement $W(L)=\left\langle(u(x)-\bar{u})^{2}\right\rangle$. We find that $g(x) \sim x^{2 \eta}$ with $\eta \simeq 3 / 4$ at short distances and $\eta \simeq 3 / 5$ at intermediate distances. We cannot resolve the asymptotic long distance dependence of $g$ upon $x$. For the system sizes considered we find $g(L / 2) \propto W \sim L^{2 \chi}$ with $\chi \simeq 2 / 3$. The exponent $\eta \simeq 3 / 5$ is in agreement with the Random Manifold exponent obtained from replica calculations and the exponent $\chi \simeq 2 / 3$ is consistent with an exact solution for the chain with transverse displacements $(u(x)$ perpendicular to $x)$. The distribution of nearest distances between pinning wells and chain-particles is found to develop forbidden regions.
\end{abstract}

PACS numbers: 74.60.Ge, 61.41.+e, 68.10,-m 


\section{Introduction}

An elastic medium in a random static potential models numerous physical phenomena such as domain walls in impure magnets, [1], flux line lattices in dirty Type II superconductors

in the mixed state, [3, 4, 5 polymers in a disordered environment, [6] etc. The configuration of the elastic medium is determined through the competition between the elastic energy of the medium and the pinning energy due to the inhomogeneities. This is a conflict between ordering and disordering. In less than four dimensions the random potential will win leading to loss of translational order at zero temperature. [3]

The degree of distortion of the elastic structure grows with the considered length scale. Two characteristics are often used to quantify the distortion. One is the displacement correlation function

$$
g(\mathbf{r})=\left\langle\left(u\left(\mathbf{r}+\mathbf{r}_{0}\right)-u\left(\mathbf{r}_{0}\right)\right)^{2}\right\rangle
$$

where $u(\mathbf{r})$ denotes the displacement $u$ of a point in the elastic medium from its ideal lattice position $\mathbf{r}$ and the angular brackets denote a thermal average, average over choice of origin, $\mathbf{r}_{0}$, and an average over the disorder.

This function is used in collective pinning theory to calculate the correlated volume, $V_{c}$ [⿴囗⿱一一), as well as in the theory of collective creep [7]. Furthermore, it has been used in attempts to calculate the correlation function of the translational order of the elastic system [8, 9].

There have been many attempts to calculate the displacement function either by use of scaling arguments (see for instance Ref. 44, 10, 7]) or using replica techniques 《11, 12, 13, 14. These studies obtain three separate regimes for $g(\mathbf{r})$. The first regime is the Larkin regime and is defined by $g(\mathbf{r})<R_{p}^{2}$, where $R_{p}$ is the length scale of the pinning potential. This short distance regime describes the behaviour within a correlated volume and is expected to be derivable from perturbation theory 4 , 7]. The second regime is often 
referred to as the Random Manifold regime, which is defined over the range $R_{p}^{2}<g(\mathbf{r})<a_{0}^{2}$, where $a_{0}$ is the ideal lattice spacing. The final regime is the asymptotic long distance regime, defined by $g(\mathbf{r})>a_{0}^{2}$. The displacement function scales differently in each of the three regimes.

There are three important dimensionalities in the problem. Firstly, there is the actual dimension of the space that the random potential occupies, $D$. Secondly there is the dimension of the components of the elastic medium, $d$ (i.e. points are $d=0$, lines $d=1$, planes $d=2$ ), and finally there is the number of dimensions perpendicular to the components of the elastic medium that the displacements move in, $n$. For example, $D=2, d=n=1$ represents an interface in a two dimensional random bond Ising model, whilst $D=3, d=2$, $n=1$ is a planar interface in three dimensions, and $D=3, d=1, n=2$ represents flux lines in dirty type II superconductors. In general, $D=d+n$. The precise scaling in each of the three regimes of $g(\mathbf{r})$ will usually depend on all three of these dimensionalities.

Another measure of the length scale dependence of the distortion is obtained from the fluctuations in the displacement field averaged over the whole system and then studied as function of different system sizes $L$. I.e.,

$$
W(L)=\left\langle(u(\mathbf{r})-\langle u\rangle)^{2}\right\rangle
$$

An exact solution for $W(L)$ was offered by Huse, Henley, and Fisher [1]. This was for an interface in the continuum version of the two dimensional random bond Ising model (i.e. $D=2, d=1, n=1$ ). They derived an exact scaling exponent for the size dependence of $W(L)$. Namely $W(L) \sim L^{2 \chi}$ with $\chi=2 / 3$. The discrete version of this model is similar to ours (discussed below) in that both consist of a chain of particles but the above model is subject to transverse displacements whilst the displacements in our chain are confined to the longitudinal direction.

Here we consider a one dimensional chain of particles connected by elastic springs 
embedded in a static random potential (i.e. $D=1, d=0, n=1$ ). Periodic as well as open boundary conditions are studied. We calculate the displacement function in Eq. 11 and the average square displacement in Eq. 20 by Monte Carlo annealing. We find that $g(x)$ exhibits huge fluctuations and that the averaged quantity is very sensitive to the specific details of the annealing and averaging procedure used. Hence, one has to be very careful in order to make sure that thermal equilibrium is achieved and that the results are reproducible.

Our motivation is to compare our results to the replica calculations of Bouchaud, Mézard and Yedidia (BMY).[11, 12] For the given dimensionalities, they obtain the following. In the Larkin regime, they obtain $g(x) \sim x^{2 \eta}$ with $\eta=3 / 2$.15 In the Random Manifold regime, they get the same scaling form as the Larkin regime but with an exponent $\eta=3 / 5$. Finally, in the long distance regime they obtain $g(x) \sim x^{2 \eta} / \sqrt{\log x}$ with $\eta=3 / 4$.

In the case of periodic boundary conditions we observe two regimes of different growth of $g(x)$. We can fit our data to an algebraic form $g(x) \sim x^{2 \eta_{i}}$. In the short distance regime $\eta_{1} \simeq 3 / 4$, in disagreement with the calculation of BMY, and in the intermediate distance regime $\eta_{2} \simeq 3 / 5$ in agreement with BMY's replica calculation. [11, 12] We cannot comment on the scaling in the long distance regime since computational restrictions prevent us from considering long enough chains.

In contrast to the theoretical expectation we find that the exponents measured in the simulation do depend on the strength of the random potential, with $\eta_{2}$ approaching $1 / 2$ for the stronger potential. This could possibly be a finite size effect.

It is very difficult to produce satisfactory statistics for the open chain. However, our simulation indicates that there is no difference between the open and the periodic chain. One might anticipate a difference, since in one case the density of particles in the chain is allowed to change whereas in the other case the density is fixed. This difference will of course be most pronounced at long distances when the relative displacement becomes of order $a_{0}$. Hence one could argue that our chains are too short to resolve the difference. Our 
largest relative displacement is of order $2 a_{0}$.

We also find that our simulations give $W(L) \sim L^{2 \chi}$, with $\chi \simeq 2 / 3$. This is consistent with the exact solution for the transverse chain. One might expect the two systems to have different exponents, but it is worth noting that for disorder free cases where the displacements are due to thermal effects only, the two systems behave with the same thermal roughening exponent of $\eta=1 / 2$. It is also worth noting that super-universal exponents can occur. Numerical simulations of Kardar and Zhang [2] show that the exponent $\chi=2 / 3$ holds for not only the exact solution where $D=2, d=1, n=1$, but also for $D=3, d=1, n=2$ and $D=4, d=1, n=3$. Finally, the difference between transverse and longitudinal displacements will be most important for relative displacements large compared to the lattice spacing. We can not exclude a cross-over to a different $\chi$-exponent at much longer distances.

In order to quantify the amount of correlation between the particles in the elastic chain and the randomly positioned pinning wells we have studied the distribution of nearest distances, $P(r)$, between particles and pinning centres. We observe that $P(r)$ develops gaps around values of the order of the range of the pinning centres. These forbidden regions vanish with increasing temperature. This behaviour can be understood in terms of an effective one particle model.

In addition to the spatial behaviour of the system we also investigate the relaxation times of the system. We have looked for the possible existence of ergodicity breaking by measuring the relaxation times as function of system size as usually done in spin glasses [17]. No ergodicity breaking was observed.

\section{The Model}

Our model consists of a one dimensional chain of $N$ harmonically coupled particles with positions $x_{i}$. The equilibrium ideal lattice spacing is $a_{0}$ and the coupling constant of the 
elastic interaction is $k$. The chain is then subjected to a static background potential created by $N_{p}$ attractive pinning wells with random positions $x_{i}^{p i n}$. The resulting Hamiltonian for the system is

$$
H=\sum_{i=1}^{N} \frac{k}{2}\left(x_{i+1}-x_{i}-a_{0}\right)^{2}+\sum_{i=1}^{N} V\left(x_{i}\right)
$$

We use $a_{0}$ as our length unit and $k$ as our energy unit, thus setting $a_{0}=k=1$. The random potential at a point $x$ is $V(x)$ and it is generated by

$$
V(x)=-\sum_{j=1}^{N_{p}} A_{p} \exp \left(-\frac{\left(x-x_{j}^{p i n}\right)^{2}}{R_{p}^{2}}\right)
$$

The pinning wells are represented by attractive Gaussians of strengths $A_{p}$ and ranges $R_{p}$. We always take the density of pinning centres as $n_{p}=N_{p} / N=1$.

We perform Monte Carlo annealing simulations on this Hamiltonian in order to study the low temperature properties of the system.

We now describe the simulation. Standard Metropolis importance sampling is used [19]. The initial configuration for the chain is chosen at random. We then choose a particle at random and give it a random displacement of $-\gamma \leq d x \leq \gamma$. The factor $\gamma$ depends on the temperature and is chosen such that the acceptance rate is kept at about $60 \%$ for all temperatures. When calculating $g(x)$ we start from a high initial temperature $T_{i}$ and anneal the system down to the final temperature $T_{f}$. We use an inversely linear time dependence of the temperature [20]

$$
T(t)=\frac{T_{i}}{t} \quad \text { for } \quad t>0
$$

The time unit in the above equation is given by $n_{\text {sweep }}$ Monte Carlo sweeps through the lattice. Thus the time unit $\Delta t=1$ corresponds to $N n_{\text {sweep }}$ Monte Carlo steps. We find that $n_{\text {sweep }}=400$ is sufficient since the results are the same for larger values of $n_{\text {sweep }}$.

At the end of the cooling we check that the simulation at $T=T_{f}$ is in equilibrium by calculating the specific heat $C=\left(\left\langle H^{2}\right\rangle-\langle H\rangle^{2}\right) / k_{B} T^{2}$ (here $k_{B}$ is Boltzmann's constant). At the very low final temperature we expect $C$ to be given by the equipartition value 
$C=N k_{B} / 2$. We then perform a thermal average of $g(x)$ by sampling the configuration of the particles every few sweeps through the lattice.

The results for $g(x)$ is supplemented by the distribution, $P(r)$, of distances $r$ between the particles in the chain and the pinning centres nearest by. This distribution contains directly information about the degree of correlation between the particle position and the positions of the pinning centres.

In addition to the calculation of $g(x)$ we also measure relaxation times in simulations performed at fixed temperature. The relaxation times are obtained from the evolution of the elastic part of the energy, $E_{e l} / N$, in Eq. 年. In these simulations we start out from a random lattice configuration. We then measure the elastic energy per particle as function of Monte Carlo time, $E_{e l}(t) / N$ [17].

\section{Results and Discussion}

Firstly, we study a system of length 200 with $A_{p}=0.01$ and $R_{p}=0.1$. The displacement function, $g(x)$, scales differently depending on whether the fluctuations in the displacement field are due to thermal roughening or disorder roughening. For a finite temperature, $T_{f}$, there is a temperature dependent crossover length, $l_{T}$ [18], that separates the two scaling regimes. For $x<l_{T}$ thermal effects dominate and $g(x)$ goes linearly in $x$. For $x>l_{T}$ disorder induced displacements dominate. Thus for any finite temperature, disorder will always dominate if long enough length scales are considered. For the considered chain, if we consider final temperatures $T_{f} \gg A_{p}$, the pinning potential becomes irrelevant since the length scale $l_{T}$ is greater than the system size and $g(x)$ is given by the exact expression for the case where there is no disorder in the system and the only fluctuations are due to temperature:

$$
g(x)=k_{B} T_{f} x\left(1-\frac{x}{N}\right)
$$


where $N=200$ is the length of the chain. For $T_{f} \ll A_{p}$, the pinning potential totally dominates, since $l_{T}$ is less than the lattice spacing, and $g(x)$ deviates from the expression in Eq. 6. If we then increase the temperature, we can clearly identify a change in the short distance behaviour towards the linear behaviour of the pure system. Unfortunately large fluctuations limit the systems sizes affordable. For this reason we have not been able to resolve the temperature dependence of $l_{T}$. Below we concentrate on the low temperature region $T \ll A_{p}$.

We show in Fig. $1 g(x)$ measured on a periodic system of length $N=200$ with $A_{p}=0.01$ and $R_{p}=0.1$. The solid curve is obtained by averaging over 600 different realisations of the random potential using the same annealing procedure for each realisation. (The dotted line is discussed below). In order for the Monte Carlo method to be able to explore as much of the phase space of the Hamiltonian as possible, we choose an initial temperature $T_{f} \gg A_{p}$ so that the disorder is irrelevant and thermal effects totally dominate in the initial stages of the simulation. The initial temperature here is $T_{i}=1$. The solid line corresponds to a final temperature of $T_{f}=10^{-3}$. We checked that an average over 800 realisations gives the same curve where as 400 relaisations were significantly different. A huge number of realisations are needed in order to obtain satisfactory statistics. This is due to the low dimensionality of the problem. Fluctuations are very significant as is seen from Fig. 2 where we have plotted a host of the $g(x)$ curves for a randomly selected subset of realisations of the random potential. These strong fluctuations are already present in the pure system (no random background potential). We studied the pure system in order to check that our annealing procedure correctly reproduces the exact result in Eq. 6 for $A_{p}=0$.

We also addressed the question of self-averaging. We did this by comparing the result obtained for $g(x)$ by averaging over many different realisations of the disorder to the result obtained for one single realisation of the disorder averaged over the initial configuration. The result for the average over initial configurations is shown as the dotted line in Fig. 
1. The final temperature is $T_{f}=10^{-3}$. The two different types of average give the same qualitative results. However, we attribute the slight difference in the curves to a finite size effect. Since the curve averaged over initial configurations is for a single realisation of 200 pinning centres, we can expect that averages of this nature will not sample the full distribution of pinning configurations as effectively as the same average for a larger system or for averages over different realisations of the disorder. Thus, we expect that the two types of averages will converge in the thermodynamic limit. I.e., the chain is self-averaging.

In Fig. 3 we show a double logarithmic plot of $g(x)$ for a periodic chain of length $N=10^{3}$ with $T_{f}=10^{-3}$ for different values of $A_{p}$ and $R_{p}$. We also show the result for an open chain. One can consistently identify two algebraic regimes where we define $x=x_{c}$ as the crossover distance and $g(x) \sim x^{2 \eta_{i}}$ in both regimes. We compare these two regimes to the Larkin regime and the Random Manifold regime of Ref. [11]. In Ref. [11] they obtain a third asymptotic regime for which $g(x)>a_{0}^{2}$, where they obtain $g(x) \sim x^{3 / 2} / \sqrt{\log x}$. It can be seen from Fig. 3 that the region $g(x)>a_{0}^{2}$ is too small to obtain any information about whether the predicted scaling in this regime is correct or not. To study this we would need to consider much longer chains which we are prevented from doing due to computational limitations. The short distance Larkin regime $x<x_{c}$ is in all four cases well described by an exponent $\eta_{1} \simeq 0.73$. This differs from the value exponent $\eta=\frac{3}{2}$ expected by BMY. [15]. The exponent $\eta_{2}$ in the Random Manifold regime $x>x_{c}$ is about 0.63 for $A_{p}=0.01$. See the solid line $\left(R_{p}=0.1\right)$ and the dashed line $\left(R_{p}=0.2\right)$. The arrow marks the value of $x_{c}$ for $A_{p}=0.01$ and $R_{p}=0.1$. It should be noted that the crossover distance $x_{c}$ always is found to be only a few lattice spacings. The deviation of this exponent from analytic expectations might be due to the inapplicability of continiuum elasticity theory at such short length scales. When $A_{p}$ is increased to 0.05 the exponent $\eta_{2}$ decreases to about 0.57 as is seen from the dot-dashed curve. These two exponents are very close the exponent $\eta=\frac{3}{5}$ as predicted in Ref. [11]. The open chain appears to behave identically to the periodic chain. See the dotted curve which describes an open chain with $A_{p}=0.01$ and $R_{p}=0.1$. The crossover 
distance $x_{c}$ is predicted to be dependent on the pinning parameters $A_{p}$ and $R_{p}$. The figure illustrates the difficulties one has in identifying any functional dependence of $x_{c}\left(A_{p}, R_{P}\right)$. It is also assumed [4, 7, 11] that $g(x)$ at the crossover distance should scale as $g\left(x_{c}\right) \sim R_{p}^{2}$. Again, it is difficult to extract concrete information about this.

It is interesting to point out that an exponent $\eta_{2}=1 / 2$ is obtained perturbatively by considering a continuum Hamiltonian with a delta-correlated random potential. 16] Consider

$$
H=\int d x\left\{\frac{1}{2} \kappa\left[\frac{d u(x)}{d x}\right]^{2}+V(x+u(x))\right\}
$$

The extrema condition $\delta H / \delta u=0$ takes the simple form

$$
0=-\kappa \frac{d^{2} u(x)}{d x^{2}}+V^{\prime}(x+u(x)) \approx-\kappa \frac{d^{2} u(x)}{d x^{2}}+V^{\prime}(x)
$$

The approximation is correct to lowest order in $V$. By integration we obtain

$$
u(x)=\frac{1}{\kappa} \int_{0}^{x} d x^{\prime} V\left(x^{\prime}\right)+u(0) .
$$

The displacement function in Eq. 11 is then given by the following pretty expression

$$
g(x)=\frac{1}{\kappa^{2}} \int_{0}^{x} d x_{1} \int_{0}^{x} d x_{2}\left\langle V\left(x_{1}\right) V\left(x_{2}\right)\right\rangle
$$

Hence, if the random potential is delta correlated $g(x) \sim x^{2 \eta}$ with $\eta=1 / 2$. We measured the correlation function of the background potential in the configurations generated by the simulation and found that the correlations decayed to zero over a very small number of springs in the chain. This decay distance was of about the same value as the crossover distance of $g(x)$ (see Fig. 1). We were unfortunately not able to identify the detailed functional form of $\left\langle V\left(x_{1}\right) V\left(x_{2}\right)\right\rangle$. This argument suggests that the exponent $\eta_{1} \approx 0.73$ measured in the short distance regime is an effect of the short distance behaviour of the effective correlations of the potential. This perturbative result might also have some bearing on the behaviour observed in the intermediate regime. At these distances the random potential can be considered delta-correlated and if Eq. 10 were applicable one should expect a value for the $\eta$ exponent of $1 / 2$. This perturbative value is not very different from the 
actually measured $\eta_{2} \approx 0.57$ to 0.63 . Nor is it very far from the replica prediction $\eta=3 / 5$. Perhaps it is appropriate to mention that perturbation theory is usually only assumed to be applicable as long as $g(x)<R_{p}^{2}$. This is probably correct when the random potential consists of a very high density $\left(N_{p} \gg N\right)$ of very sharp pinning centres. In the case we have simulated $N_{p}=N$. Only few particles are positioned in the steep regions of the pinning wells where a displacement of order $R_{p}$ produces significant changes in $V(x+u(x))$. Hence, the approximation used in Eq. 8 might still be reasonable for most of the particles even when $g(x)$ becomes equal to or somewhat larger than $R_{p}^{2}$.

We show in Fig. 4 a double logarithmic plot of $g(L / 2)$, together with $W(L)$ (see Eq. 2) for parameters $A_{p}=0.05, R_{p}=0.1$ and $n_{p}=1$. The two solid lines have both slope $4 / 3$. One sees that the behaviour of both quantities are consistent with the exact result for the transverse chain $W(L) \sim L^{4 / 3}$. [1] However, one notices also that the data points at $L=10^{3}$ fall below the straight line. This might indicate a crossover to a different behaviour at longer distances. Hence, we cannot exclude that the asymptotic behaviour of $W$ and $g(L / 2)$ for the considered longitudinal displacements might be different from the exactly solvable transverse case.

The behaviour of $g(x)$ is put in perspective by the results for the distribution of nearest distances between particles and pinning centres shown in Fig.5. Either side of the sharp central peak there is a dip in the distribution showing the existence of a 'forbidden region' were the particles are less likely to sit. These regions disappear in the high temperature phase (i.e. when $l_{T}>N$ ) were the distribution is slightly peaked at the centre and decreases monotonically as $r$ increases. We can account for the forbidden regions by considering the following simple model. We describe a single particle in the chain and the pinning centre nearest to it by the following model Hamiltonian

$$
\begin{array}{ll}
H=\frac{k_{e f f}}{2}\left(x-x_{0}\right)^{2}+A_{p}\left(\left(\frac{x}{R_{p}}\right)^{2}-1\right) & |x|<R_{p} \\
H=\frac{k_{e f f}}{2}\left(x-x_{0}\right)^{2} & |x|>R_{p}
\end{array}
$$

Thus we consider the particle to be interacting with a potential well of $\operatorname{depth} A_{p}$, centred 
at $x=0$ and of range $R_{p}$, and that this particle is coupled harmonically to the rest of the chain with an effective coupling constant $k_{e f f}$ and an elastic energy zero at position $x_{0}$. The system is restricted in size to $-a \leq\left(x, x_{0}\right) \leq a$, where the parameter $a$ is given by the density of pinning centres in the simulations $2 a=\frac{1}{n_{p}}$. Since for each particle the parameter $x_{0}$ will differ, when we calculate the distribution from the simulations, this is effectively the same as averaging the above Hamiltonian (or the probability function $e^{-\beta H}$ ) over $x_{0}$. Thus, we want to calculate

$$
\langle P(x)\rangle_{x_{0}}=\frac{1}{2 a} \int_{-a}^{a} \frac{e^{-\beta H\left(x, x_{0}\right)}}{Z\left(x_{0}, \beta\right)} d x_{0}
$$

where $Z\left(x_{0}, \beta\right)$ is the usual canonical partition function with the exception that the $x$ integration is over the finite interval $-a \leq x \leq a$. As usual $\beta$ is the inverse temperature. The calculation reduces to a sum of error functions and the $x_{0}$ integration has to be done numerically. The effective spring constant will be $k_{\text {eff }}=\alpha k$, where $1 / \alpha$ gives the number of springs which are affected by the displacement of a single particle [21, 22].

Fig. 5. shows the distribution $P(r)$ obtained from a simulation with parameters $N=10^{3}, A_{p}=0.01, R_{p}=0.1, n_{p}=1$ and $\beta=10^{3}$. The inset to Fig. 5. shows the calculated distribution $\langle P(x)\rangle_{x_{0}}$ with the same parameters as the simulation and with a renormalisation constant $\alpha=0.175$. The forbidden regions can be seen clearly in both cases. It is captivating to notice that the length scale $1 / \alpha=5.7$ is identical to the length scale $x_{c}$ at which $g(x)$ crosses between the two different algebraic regimes for this set of parameters. See Fig. 3 where the arrow at the $\mathrm{x}$-axis locates the value $\log _{10}(1 / \alpha)$.

It is of interest to measure the relaxation times of the system in order to decide upon the possibility of ergodicity, or replica, breaking in the disordered chain [17]. We measured the time dependence of the elastic energy $E_{e l}(t)$ (given by the first term in the Hamiltonian in Eq. 31). The relaxation of $E_{e l}(t)$ for different chain lengths is exhibited in Fig. 6 for $A_{p}=0.01, R_{p}=0.1$ at a temperature of $T=10^{-3}$. An average over different realisations of the random potential has been performed. The temporal behaviour of $E_{e l}(t)$ is independent of the length of the chain. Hence, we conclude that the disorder is unable to break the 
ergodicity of the system.

\section{Conclusion}

We have studied a harmonic chain at low temperature subject to a random potential. Fluctuations, both thermal as well as disorder, are very significant. This puts unpleasant limits on the system sizes which one can simulate in a reliable way. In spite of these limitations we conclude the following concerning the spatial behaviour (at low temperatures) of the displacement function $g(x)$. A crossover length $x_{c}$ exists. For $x<x_{c}$ we find $g(x) \sim x^{2 \eta_{1}}$ with $\eta_{1} \simeq 3 / 4$, in disagreement with the expected Larkin exponent. For $x>x_{c}$ we find $g(x) \sim x^{2 \eta_{2}}$ where $\eta_{2} \simeq 3 / 5$, in agreement with replica theory. We are not able to identify the functional dependence of $x_{c}$ on the strength and range of the randomly positioned pinning wells. We do not find any difference in the behaviour of the open and the periodic chain. We are also unable to consider chains of sufficient length so to study the appearance of a third scaling region for $g(x)>a_{0}^{2}$.

The average square fluctuations $W(L) \sim L^{2 \chi}$ was found to depend on the length of the chain with an exponent $\chi$ consistent with the exact result for a transverse chain $\chi=2 / 3$. The exponent might change on length scales beyond our reach.

We studied the distribution $P(r)$ of nearest distance between particles and pinning wells. As the temperature is lowered (or $A_{p}$ is increased) particles become prohibited from a region around $R_{p}$ away from the centre of the pinning wells.

The crossover distance $x_{c}$ and the distribution $P(r)$ can be connected. In an effective single particle model of $P(r)$ the scale $x_{c}$ reappears as the ratio between the effective spring constant and the bare spring constant.

The system was found to be self-averaging. A study of relaxation times in the system 
showed that (as expected in one dimension) no ergodicity breaking occur.

Acknowledgement: We are grateful to J.P. Bouchaud, K.H. Fischer, P. Holdsworth, and D. O'Kane for stimulating discussions. The work is supported by the British EPSRC and DRA Malvern. 


\section{References}

[1] D.A. Huse, C.L. Henley, and D. Fisher, Phys. Rev. Lett. 55, 2924 (1985), M. Kardar, ibid. 55, 2923 (1985), D. Huse, C.A. Henley, and D. Fisher, ibid. 55, 2924 (1985).

[2] M. Kardar and Y. -C. Zhang, Phys. Rev. Lett. 58, 2087 (1987).

[3] A. I. Larkin, Sov. Phys. JETP 31784 (1970)

[4] A. I. Larkin and Yu. N. Ovchinnikov, J. Low Temp. Phys. 34409 (1979).

[5] G. Blatter, M.V. Feigel'man, V.B. Geshkenbein, A.I. Larkin, and V.M. Vinokur, Rev. Mod. Phys. in press.

[6] See for instance S.F. Edwards and M. Muthukumar, J. Chem. Phys. 89, 2435 (1988), and references therein.

[7] M. Feigel'man, V. Geshkenbein, A. I. Larkin and V. M. Vinokur, Phys. Rev. Lett. 63 $2303(1989)$

[8] E.M. Chudnovsky Phys. Rev. B 43, 7831 (1991).

[9] A. Houghton, R.A. Pelcovits, A. Sudbø, J. of Phys. Condens Matter 3, 7527 (1991).

[10] T. Natterman, Phys. Rev. Lett. 642454 (1990).

[11] J.-P. Bouchaud, M. Mézard and J.S. Yedidia, Phys. Rev. B 4614686 (1992).

[12] J.-P. Bouchaud, M. Mézard and J.S. Yedidia, Phys. Rev. Lett. 673840 (1991).

[13] T. Giamarchi and P. Le Doussal, Phys. Rev. Lett. 721530 (1994).

[14] S. E. Korshunov, Phys. Rev. B 483969 (1993).

[15] The Larkin exponent of $\eta=\frac{3}{2}$ is obtained from a random force model. This exponent is now believed to be $\eta=1$. J. -P. Bouchaud, private communication. 
[16] Delta correlated random forces produces in perturbation theory an exponent $\eta=3 / 2$.

[17] N.D. Mackenzie and A.P. Young, Phys. Rev. Lett. 49301 (1982) and J. Phys. C 16, $5321(1984)$.

[18] See Kardar in Ref. [1].

[19] J.J. Binney, N.J. Dowrick, A.J. Fisher, and M.E.J. Newman The Theory of Critical Phenomena, Oxford Press 1992.

[20] H. Szu and R. Hartley, Phys. Lett. A 122157 (1987).

[21] The idea is the following. Take $n$ springs each of spring constant $k$. Couple these $n$ springs in a series. The effective spring constant $k_{e f f}$ of the series is $k_{e f f}=k / n$.

[22] Elastic instabilities at zero temperature leads also to inaccessible regions in the random potential, see H.J. Jensen, Y. Brechet, B. Doucot, and A. Brass, Europhys. Lett. 23, $623(1993)$. 


\section{Captions}

\section{Figure 1 .}

The displacement correlation function. Solid line corresponds to an average over 600 realisations of the random potential. The dotted line is generated by an average over 2300 different initial starting configurations all annealed down into the same realisation of the random potential. Parameters are $N=200, A_{p}=0.01, R_{p}=0.1, n_{p}=1, T_{i}=1$, and $T_{f}=10^{-3}$.

\section{Figure 2.}

A host of the concrete realisations of $g(x)$ entering into the averaged $g(x)$ shown as the solid line in Fig. 1.

\section{Figure 3.}

Double logarithmic plot of $g(x)$ in four different cases. Solid line represents $A_{p}=0.01$ and $R_{p}=0.1$. Dashed line corresponds to $A_{p}=0.01$ and $R_{p}=0.2$. The dot-dashed line belongs to $A_{p}=0.05$ and $R_{p}=0.1$. These three curves are for periodic chains. The dotted curve represents the measurement on an open chain with $A_{p}=0.01$ and $R_{p}=0.1$. In all cases $N=10^{3}, T_{i}=1$, and $T_{f}=10^{-3}$. The arrow indicates the location of $\log _{10}\left(x_{c}\right)$ which coincides with the location of $\log _{10}(1 / \alpha)$. See the text for an explanation.

\section{Figure 4.}

Double logarithmic plot of the average fluctuations in the displacement $W(L)$ as function of $L$ (crosses) together with the amplitude $g(L / 2)$ of the displacement correlation function (circles).

\section{Figure 5.}

The distribution of nearest distance between particles in the chain and pinning centres. Parameters are $A_{p}=0.01$ for the solid line and $A_{p}=0.05$ for the dashed curve. In both cases $R_{p}=0.1, n_{p}=1, N=10^{3}, T_{i}=1$, and $T_{f}=10^{-3}$. The insert corresponds to the solid 
curve. It is calculated from the single particle model with a scaling paramenter $\alpha=0.175$.

\section{Figure 6.}

The time dependence of the elastic energy (measured per particle). The simulation is run at fixed temperature $T=10^{-3}$. Curves for $N=200,300,400$, and 800 are shown. In all cases $A_{p}=0.01, R_{p}=0.1, n_{p}=1$. 